\title{
R0S generated by CYP450, especially CYP2E1, mediate mitochondrial dysfunction induced by tetrandrine in rat hepatocytes
}

\author{
Xin-ming QI", Ling-ling MIAO*, Yan CAI, Li-kun GONG* , Jin REN* \\ Center for Drug Safety Evaluation and Research, Shanghai Institute of Materia Medica, Chinese Academy of Sciences, Shanghai \\ 201203, China
}

\begin{abstract}
Aim: Tetrandrine, an alkaloid with a remarkable pharmacological profile, induces oxidative stress and mitochondrial dysfunction in hepatocytes; however, mitochondria are not the direct target of tetrandrine, which prompts us to elucidate the role of oxidative stress in tetrandrine-induced mitochondrial dysfunction and the sources of oxidative stress.

Methods: Rat primary hepatocytes were isolated by two-step collagenase perfusion. Mitochondrial function was evaluated by analyzing ATP content, mitochondrial membrane potential (MMP) and the mitochondrial permeability transition. The oxidative stress was evaluated by examining changes in the levels of reactive oxygen species (ROS) and glutathione (GSH).

Results: ROS scavengers largely attenuated the cytotoxicity induced by tetrandrine in rat hepatocytes, indicating the important role of ROS in the hepatotoxicity of tetrandrine. Of the multiple ROS inhibitors that were tested, only inhibitors of CYP450 (SKF-525A and others) reduced the ROS levels and ameliorated the depletion of GSH. Mitochondrial function assays showed that the mitochondrial permeability transition (MPT) induced by tetrandrine was inhibited by SKF-525A and vitamin C (VC), both of which also rescued the depletion of ATP levels and the mitochondrial membrane potential. Upon inhibiting specific CYP450 isoforms, we observed that the inhibitors of CYP2D, CYP2C, and CYP2E1 attenuated the ATP depletion that occurred following tetrandrine exposure, whereas the inhibitors of CYP2D and CYP2E1 reduced the ROS induced by tetrandrine. Overexpression of CYP2E1 enhanced the tetrandrineinduced cytotoxicity.

Conclusion: We demonstrated that CYP450 plays an important role in the mitochondrial dysfunction induced by the administration of tetrandrine. ROS generated by CYP450, especially CYP2E1, may contribute to the mitochondrial dysfunction induced by tetrandrine.
\end{abstract}

Keywords: tetrandrine; mitochondria; reactive oxygen species; cytochrome P450

Acta Pharmacologica Sinica (2013) 34: 1229-1236; doi: 10.1038/aps.2013.62; published online 29 Jul 2013

\section{Introduction}

Tetrandrine (Tet, Figure 1) is a compound extracted from the root of Stephania tetrandra and is often used for the treatment of rheumatism, inflammation, occlusive cardiovascular disorders and tumors ${ }^{[1-8]}$. Some early studies demonstrated that tetrandrine causes hepatotoxicity ${ }^{[9,10]}$.

Mitochondrial dysfunction is involved in many diseases and drug-induced toxicities. In our previous work, we showed that tetrandrine causes hepatotoxicity both in vivo and in vitro, and mitochondrial dysfunction plays an important role in mediating this process. Tetrandrine-induced mitochondrial dysfunction is cyclosporine A-sensitive, suggesting the pres-

\footnotetext{
\# These authors contributed equally to this study.

* To whom correspondence should be addressed.

E-mail Ikgong@cdser.simm.ac.cn (Li-kun GONG); jren@cdser.simm.ac.cn (Jin REN)

Received 2013-01-29 Accepted 2013-04-22
}

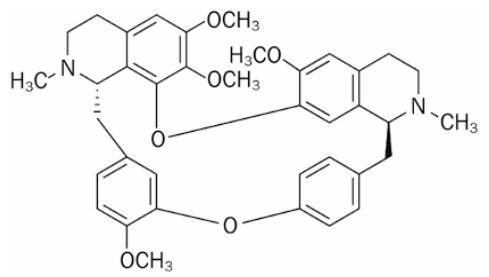

Figure 1. Structure of tetrandrine.

ence of a mitochondrial permeability transition $(\mathrm{MPT})^{[7,9,11]}$. The MPT plays a major role in mitochondrial dysfunction and represents an abrupt increase in the permeability of the mitochondrial inner membrane, resulting in the decrease of cellular ATP content and the dissipation of mitochondrial membrane potential. The MPT can be specifically inhibited by cyclosporine A $(\mathrm{CsA})^{[12-14]}$. 
Another study showed that tetrandrine could protect mitochondria isolated from rat livers against the damage induced by oxidants ${ }^{[15]}$. These two inconsistent reports indicated that mitochondrial dysfunction induced by tetrandrine in hepatocytes may be not due to the direct action of tetrandrine on mitochondria, but rather by other mechanisms that indirectly influence tetrandrine-induced mitochondrial damage.

MPT can be induced by endogenous ROS that are generated by the metabolism of drugs, pollutants and chemicals ${ }^{[16-18]}$. The increase of ROS induced by tetrandrine has been previously reported $^{[9,19]}$. A recent study showed that ketoconazole, an inhibitor of CYP3A, protects mice against pulmonary toxicity induced by tetrandrine ${ }^{[20]}$. These studies suggested a potential role of CYP450 in ROS generation and MPT induced by tetrandrine. Therefore, we designed the present study to study the role of ROS and CYP450 in tetrandrine-induced mitochondrial dysfunction using rat primary hepatocytes as a model system for hepatotoxicity.

\section{Materials and methods}

\section{Reagents and cell culture}

Tetrandrine (Sigma Chem Co, St Louis, MO, USA) was dissolved in $50 \mu \mathrm{L}$ of $0.1 \mathrm{~mol} / \mathrm{L} \mathrm{HCl}$ and diluted with $\mathrm{HBS}$ solution $(33 \mathrm{mmol} / \mathrm{L}$ HEPES, $160.8 \mathrm{mmol} / \mathrm{L} \mathrm{NaCl}, 3.15 \mathrm{mmol} / \mathrm{L}$ $\mathrm{KCl}, 0.7 \mathrm{mmol} / \mathrm{L} \mathrm{Na}_{2} \mathrm{HPO}_{4} \cdot 12 \mathrm{H}_{2} \mathrm{O}$, pH 7.65) to a final concentration of $10 \mathrm{mmol} / \mathrm{L}$.

Hepatocytes were isolated from Sprague-Dawley male rats (180-220 g) by two-step collagenase perfusion as previously described with some modifications ${ }^{[21-23]}$. Collagenase IV was purchased from Sigma Chem Co (St Louis, MO, USA). Hepatocytes were cultured as a monolayer in Ham's F-12/DMEM (Invitrogen, Carlsbad, CA, USA) (1:1) medium supplemented with $15 \%$ fetal bovine serum (PAA Laboratories GmbH, Linz, Austria).

HL-7702 cells, an immortalized hepatocyte cell line derived from a human liver (Cell Bank, SIBS, CAS, Shanghai, China), were cultured in DMEM containing 10\% fetal bovine serum (PAA Laboratories GmbH, Linz, Austria), $100 \mathrm{U} / \mathrm{mL}$ penicillin and $70 \mu \mathrm{g} / \mathrm{mL}$ streptomycin.

\section{Plasmid construction and transfection}

The coding sequences of human CYP2C8 (NM_000770, 96-1568), CYP2D6 (NM_000106, 91-1584) and CYP2E1 (NM_000773, 34-1515) were amplified by high fidelity PCR and cloned into the pcDNA3.1 (-) plasmid (Invitrogen, Carlsbad, CA, USA). The following primers were used for cloning: CYP2C8 (Xho I/Hind III, Forward: 5'-CCCCTCGAGGCCACCATGGAACCTTTTGTGGTCCTG-3', Reverse: 5'-CCCAAGCTTTCAGACAGGGATGAAGCAGA-3'), CYP2D6 (BamH I/Hind III, Forward: 5'-CGGGATCCGCCACCATGGGGCTAGAAGCACTGGT-3', Reverse: 5'-CCCAAGCTTCTAGCGGGGCACAGCACAAA-3') and CYP2E1 (EcoR I/Hind III, Forward: 5'-CCCGAATTCGCCACCATGTCTGCCCTCGGAGTCAC-3', Reverse: 5'-CCCAAGCTTTCATGAGCGGGGAATGACAC-3'). HL-7702 cells were seeded at $5 \times 10^{5}$ cells $/ \mathrm{mL}$ per well in 6 -well plates in growth medium containing $10 \%$ serum. Cells were grown for $24 \mathrm{~h}$ at $37^{\circ} \mathrm{C}$ and transfected with plasmids using Lipofectamine 2000 (Invitrogen, Carlsbad, CA, USA) according to the manufacturer's instructions. The expression of CYP450 protein was determined by Western blotting $48 \mathrm{~h}$ after transfection.

\section{Cell viability and ATP bioluminescence assays}

Cell viability and ATP bioluminescence reagents were provided in the CellTiter-Glo ${ }^{\mathrm{TM}}$ Luminescent Cell Viability Assay kit (Promega, Madison, WI, USA). Intracellular ATP levels were determined according to the manufacturer's protocol. After a 24-h treatment with tetrandrine, the bioluminescence was measured by a fluorimeter (NOVOstar, BMG LABTECH, Offenburg, Germany).

\section{Measurement of the mitochondrial membrane potential}

The mitochondrial membrane potential was measured by the accumulation of rhodamine 123 (Sigma Chem Co) according to a previously published method ${ }^{[24]}$. After treatment with tetrandrine $(25 \mu \mathrm{mol} / \mathrm{L})$ for either $4 \mathrm{~h}$ or $8 \mathrm{~h}$, the hepatocytes were washed twice with PBS and loaded with rhodamine 123 $(1 \mu \mathrm{mol} / \mathrm{L})$ for $20 \mathrm{~min}$. The fluorescence was detected on the NOVOstar at an excitation of $485 \mathrm{~nm}$ and emission of $520 \mathrm{~nm}$.

\section{Measurement of ROS generation}

DCFH-DA (Sigma Chem Co, St Louis, MO, USA) was used to detect the intracellular generation of ROS by tetrandrine as previously described ${ }^{[25]}$. After a 1-h treatment with tetrandrine (25 $\mu \mathrm{mol} / \mathrm{L})$, rat primary hepatocytes were washed twice and resuspended in PBS $\left(1 \times 10^{6}\right.$ cells $\left./ \mathrm{mL}\right)$. This cell suspension was loaded with DCFH-DA $(5 \mu \mathrm{mol} / \mathrm{L})$ and incubated at $37^{\circ} \mathrm{C}$ for $20 \mathrm{~min}$. The fluorescence of DCFH was measured on NOVOstar at an excitation of $485 \mathrm{~nm}$ and emission of $520 \mathrm{~nm}$.

\section{Measurement of intracellular GSH content}

GSH levels in the hepatocytes were measured according to the method described by Dringen and Hamprecht with some modifications ${ }^{[26]}$. Cell homogenates were diluted with $10 \%$ trichloroacetic acid and centrifuged at $3000 \times g$ for $5 \mathrm{~min}$. The

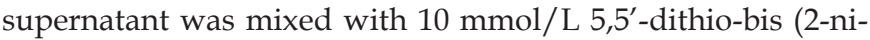
trobenzoic acid) (DTNB) and the absorbance was measured at $412 \mathrm{~nm}$ within $5 \mathrm{~min}$. Reduced pure GSH was used to generate a standard curve.

\section{Measurement of caspase 3 activity}

Caspase 3 activity was measured with a caspase 3 assay kit (BD Biosciences Pharmingen, San Diego, CA, USA). Reaction mixtures containing Ac-DEVD-AMC and cell lysates in buffer were incubated for $1 \mathrm{~h}$ at $37^{\circ} \mathrm{C}$. AMC liberated from AcDEVD-AMC was measured in the NOVOstar at an excitation of $380 \mathrm{~nm}$ and emission of $460 \mathrm{~nm}$.

Confocal microscopy and detection of the mitochondrial permeability transition

Cell loading and confocal microscopy were performed as previously described ${ }^{[27]}$. Briefly, $5 \times 10^{6}$ hepatocytes were plated 
on $40-\mathrm{mm}$ diameter glass coverslips and loaded with 500 nmol/L TMRE for $30 \mathrm{~min}$ at $37^{\circ} \mathrm{C}$. After three washes with KRH buffer, the coverslips were mounted on a Leica TCS-SP2 laser scanning confocal microscope. To reduce the influence of room temperature, an increased tetrandrine concentration (50 $\mathrm{mmol} / \mathrm{L})$ and shorter incubation time (30 min) were used. Images were collected at the indicated time points after adding tetrandrine in KRH buffer containing $100 \mathrm{nmol} / \mathrm{L}$ TMRE. The occurrence of the MPT was defined as loss of bright mitochondrial TMRE staining, which is indicative of mitochondrial depolarization. During the incubation, the hepatocytes of the control group did not show any loss of TMRE staining.

\section{Western blotting}

Standard Western blotting analysis was performed using polyclonal antibodies against $\beta$-actin, CYP2C8, CYP2D6, and CYP2E1 (Millipore, Billerica, MA, USA).

\section{Statistical analysis}

Data were entered into a database and analyzed using SPSS software. Group mean values and standard deviations were calculated. After homogeneic analysis, homogeneous data were analyzed using one-way analysis of variance and a post hoc test of least significant difference. Heterogeneous data were analyzed using $t$-tests. When $P<0.05$, the results were considered to be statistically significant.

\section{Results}

The protective effects of ROS scavengers on the cytotoxicity induced by tetrandrine in rat hepatocytes

In our previous work, we reported an increase of ROS induced by tetrandrine treatment in rat hepatocytes ${ }^{[9]}$. The role of oxidative damage in the hepatotoxicity of tetrandrine was examined by using three ROS scavengers (NAC, VE, and VC), all of which largely attenuated the cytotoxicity of tetrandrine (25 $\mu \mathrm{mol} / \mathrm{L}$ ) at $8 \mathrm{~h}$ (Figure 2).

The protective effects of CYP450 inhibitors on oxidative stress induced by tetrandrine

To analyze the source of the induced ROS, different inhibitors of ROS generation that block several different intracellular ROS-producing systems were applied. The inhibitors used are

Table 1. Different enzyme inhibitors on ROS-producing systems.

\begin{tabular}{llc}
\hline ROS production system & \multicolumn{1}{c}{ Compound } & Concentration \\
\hline Flavoproteins & $\begin{array}{c}\text { Diphenyleneiodonium- } \\
\text { chloride (DPI) }\end{array}$ & $5 \mu \mathrm{mol} / \mathrm{L}$ \\
Mitochondrial complex I & Rotenone (ROT) & $100 \mathrm{nmol} / \mathrm{L}$ \\
ATP synthase & Oligomycin (OLM) & $0.2 \mathrm{mg} / \mathrm{mL}$ \\
Cyclooxygenase & Indomethacin (IMT) & $100 \mu \mathrm{mol} / \mathrm{L}$ \\
Cytochrome P450 & Proadifen (SKF-525A, SKF) & $10 \mu \mathrm{mol} / \mathrm{L}$ \\
& Metyrapone (MET) & $500 \mu \mathrm{mol} / \mathrm{L}$ \\
& 1-Aminobenzotriazole (ABT) & $1 \mathrm{mmol} / \mathrm{L}$ \\
Xanthine oxidase & Allopurinol (ALP) & $100 \mu \mathrm{mol} / \mathrm{L}$ \\
\hline
\end{tabular}
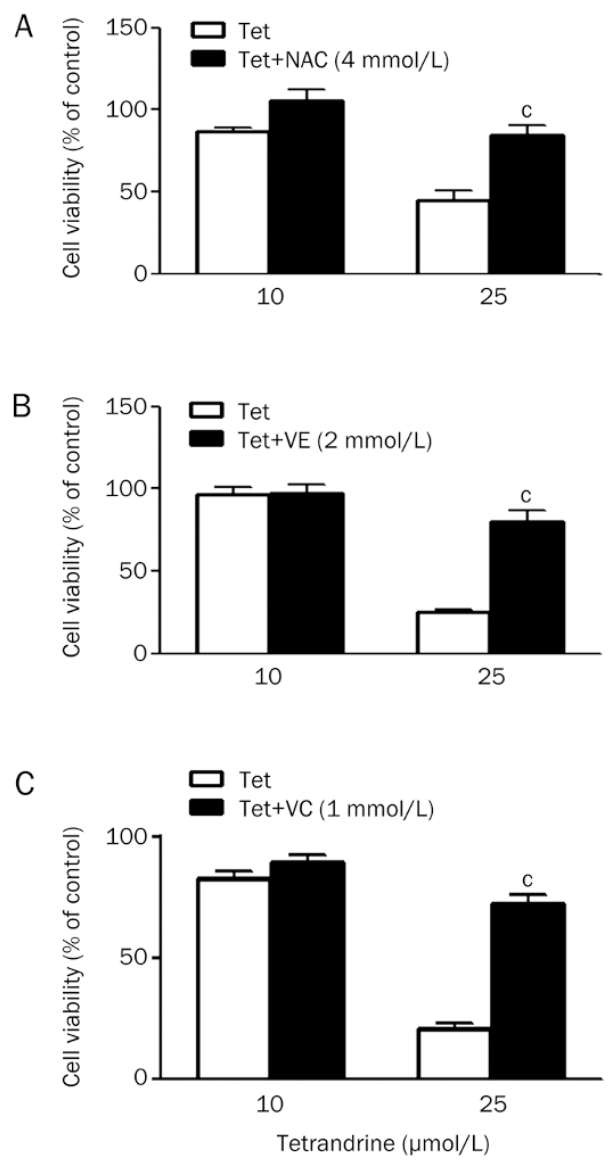

Figure 2. Protective effect of ROS scavengers on the cytotoxicity of tetrandrine (10 and $25 \mu \mathrm{mol} / \mathrm{L}$ ) after 8 -h treatment in rat primary hepatocytes. Cell viability was evaluated by ATP content. (A) Effect of N-acetylcysteine (NAC, $4 \mathrm{mmol} / \mathrm{L}$ ) on the cytotoxicity of tetrandrine. (B) Effect of vitamin $E(V E, 2 \mathrm{mmol} / \mathrm{L})$ on the cytotoxicity of tetrandrine. (C) Effect of vitamin C (VC, $1 \mathrm{mmol} / \mathrm{L})$ on the cytotoxicity of tetrandrine. All of the results are presented as the mean \pm SD. $n=3 .{ }^{~} P<0.01$ vs 'Tet' group.

summarized in Table 1. As shown in Figure 3A and Figure S1, only inhibitors of CYP450 blocked the increase of DCFH fluorescence after a 1-h treatment with tetrandrine.

Additional assays were performed to examine the effect of the CYP450 inhibitor SKF-525A (SKF) on cellular GSH content after treatment with tetrandrine for either $4 \mathrm{~h}$ or $8 \mathrm{~h}$. Depleted GSH levels were attenuated by SKF (Figure 3B). No protection was observed when using inhibitors of other ROS-producing systems.

The CYP450 inhibitor SKF and ROS scavenger VC recovered the mitochondrial dysfunction induced by tetrandrine

TMRE, a cationic fluorophore, accumulates in the mitochondria of cultured hepatocytes in response to a negative mitochondrial membrane potential. During normal aerobic incubation, confocal microscopy reveals the mitochondria as bright red spots, which is consistent with previous results ${ }^{[28]}$.

After $30 \mathrm{~min}$ of exposure to tetrandrine $(50 \mu \mathrm{mol} / \mathrm{L})$, more than 80 percent of the mitochondria lost TMRE fluorescence, 

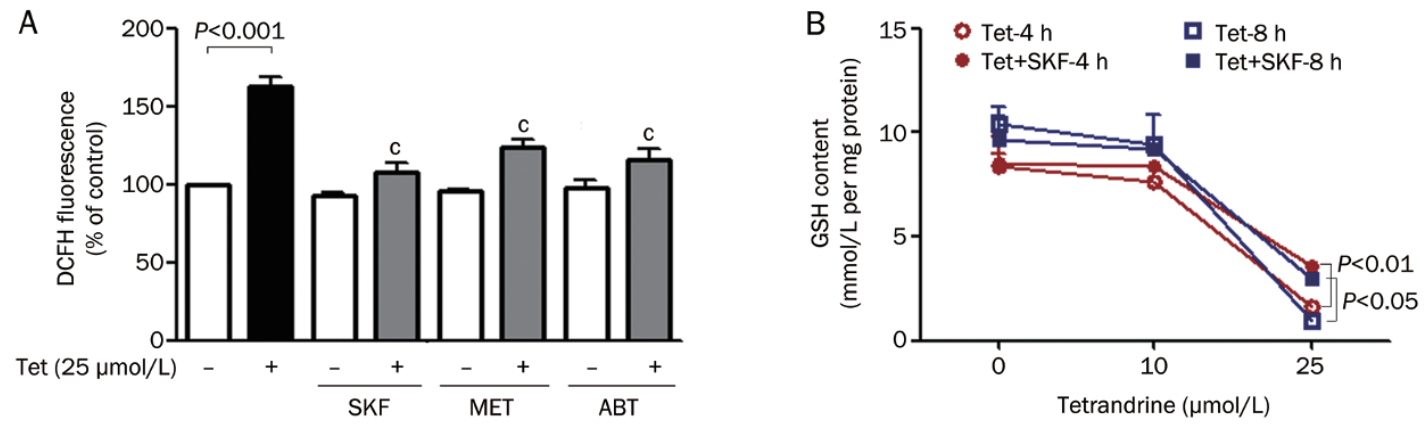

Figure 3. Protective effect of CYP450 inhibitors on oxidative stress induced by tetrandrine. (A) Effects of CYP450 inhibitors on ROS production induced by tetrandrine. ROS were detected using the fluorescent probe DCFH-DA. Rat hepatocytes were incubated with $25 \mu \mathrm{mol} / \mathrm{L}$ Tet and the indicated inhibitors for $1 \mathrm{~h}$. The concentration of each inhibitor is listed in Table 1. (B) Effect of SKF on the depletion of GSH induced by tetrandrine (10 and 25 $\mu \mathrm{mol} / \mathrm{L})$. GSH content was measured in rat primary hepatocytes at time points up to $8 \mathrm{~h}$ in either the presence or absence of SKF (10 $\mu \mathrm{mol} / \mathrm{L})$. All of the results are presented as the mean \pm SD. $n=4 .{ }^{~} P<0.01$ vs 'Tet $25 \mu \mathrm{mol} / \mathrm{L}$ ' group.

indicating depolarization of mitochondria and the presence of the MPT (Figure 4A1-4A3). We further examined the effect of VC and SKF-525A (SKF) on tetrandrine-induced MPT. When cotreated with VC (1 mmol/L), approximately two-thirds of all of the mitochondria maintained TMRE fluorescence (Figure $4 C 1-4 C 3,4 D)$. In the presence of SKF, approximately $20 \%$ of mitochondria had undergone MPT and a change in the distribution of TMRE (Figure 4B1-4B3, 4D).

Next, we tested the ability of VC and SKF to protect against tetrandrine-induced depletion of ATP and mitochondrial membrane potential (MMP) ${ }^{[9]}$. VC and SKF both significantly inhibited tetrandrine-induced ATP and MMP depletion, with SKF exerting a stronger protective effect than VC and CsA (Figure 5A, 5B; Figure S2).

The increased permeability of the mitochondrial inner membrane allows for the release of cytochrome $c$ into the cytosol, which activates downstream apoptotic factors such as caspase $3^{[14]}$. Tetrandrine induced the release of cytochrome $c$ and
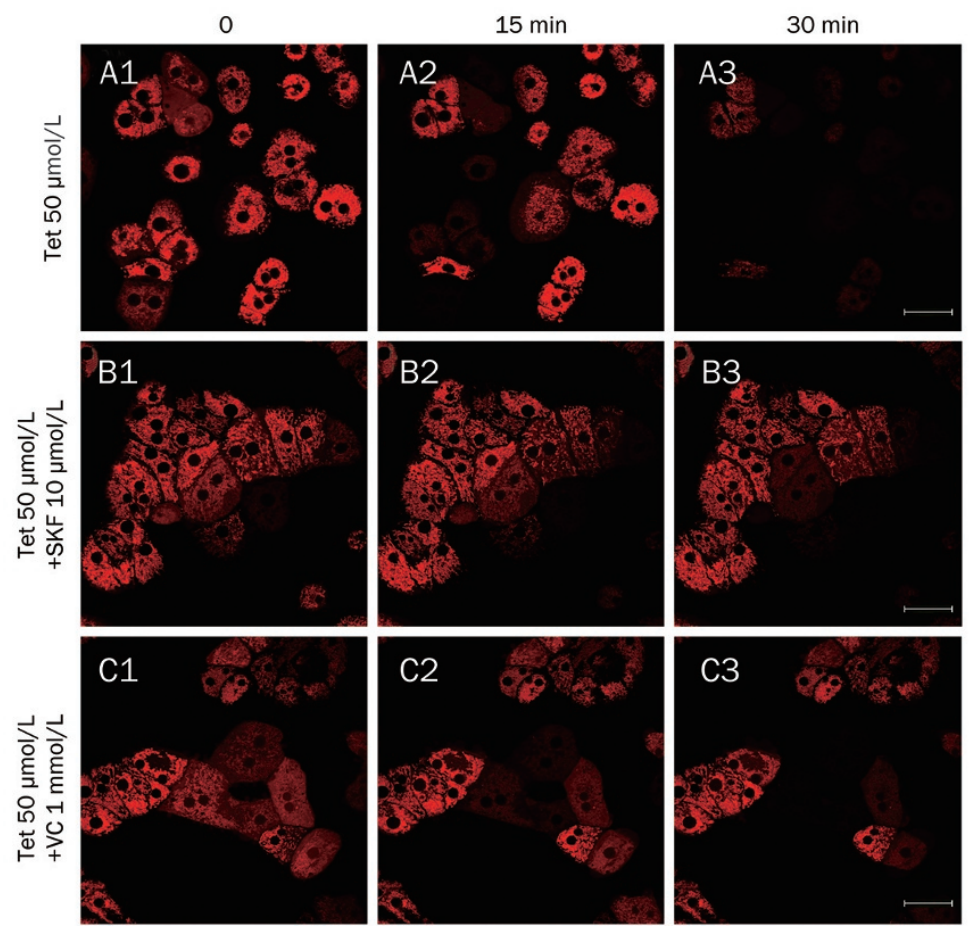

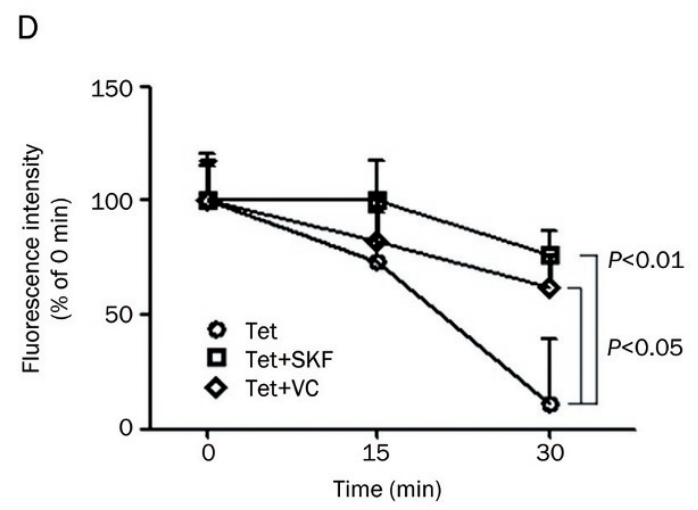

Figure 4. Effect of SKF and VC on the mitochondrial permeability transition induced by tetrandrine. TMRE accumulates into the mitochondria. A loss of the red fluorescence characteristic of TMRE indicates the depolarization of mitochondria and the presence of the mitochondrial permeability transition. Rat hepatocytes were incubated with $50 \mu \mathrm{mol} / \mathrm{L}$ Tet alone (A1-A3), or in combination with either $10 \mu \mathrm{mol} / \mathrm{L} \mathrm{SKF}$ (B1-B3) or $1 \mathrm{mmol} / \mathrm{L}$ VC (C1-C3) for $30 \mathrm{~min}$. The scale bar is $47.62 \mu \mathrm{m}$. (D) Red fluorescence intensity was semi-quantitated from confocal images in three separated experiments. SKF, SKF-525A, a nonspecific inhibitor of CYP450. All of the results are presented as the mean \pm SD. $n=3$. 
activated caspase $3^{[9]}$. Treatment with SKF showed a marked inhibition of caspase 3 activity induced by tetrandrine (Figure 5C).

Role of CYP2E1 in the oxidative stress and cytotoxicity induced by tetrandrine

Multiple inhibitors (Table 2) for the rat CYP450 isoforms were used to examine which CYP450 isoforms may be involved in the oxidative stress and cytotoxicity induced by tetrandrine. The inhibitors of CYP2C (sulfaphenazole, SFZ), CYP2D (quinine, QUN), and CYP2E1 (diallyl sulfide, DAS) significantly reduced tetrandrine-induced ROS production (Figure 6A \& Figure S3A). These inhibitors also attenuated the ATP depletion induced by tetrandrine (Figure 6B and Figure S3B).

Table 2. Inhibitors of CYP450 isoforms.

\begin{tabular}{llr}
\hline Rat CYP450 isoforms & \multicolumn{1}{c}{ Inhibitor } & Concentration \\
\hline CYP1A1, CYP1A2 & Alpha-naphthoflavone (ANF) & $5 \mu \mathrm{mol} / \mathrm{L}$ \\
CYP2C6, CYP2C11 & Sulfaphenazole (SFZ) & $20 \mu \mathrm{mol} / \mathrm{L}$ \\
& Cimetidine (CMD) & $50 \mu \mathrm{mol} / \mathrm{L}$ \\
CYP2D1, CYP2D2 & Quinine (QUN) & $20 \mu \mathrm{mol} / \mathrm{L}$ \\
CYP2E1 & Diallylsulfide (DAS) & $2 \mathrm{mmol} / \mathrm{L}$ \\
CYP3A1, CYP3A2 & Ketoconazole (KCZ) & $5 \mu \mathrm{mol} / \mathrm{L}$ \\
& Trioleandomycin (TAO) & $10 \mu \mathrm{mol} / \mathrm{L}$ \\
CYP4A1 & 10-Undecynoic acid (UDA) & $1 \mathrm{mmol} / \mathrm{L}$ \\
\hline
\end{tabular}

We constructed plasmids containing human CYP2C8, CYP2D6 and CYP2E1 and transiently transfected them individually into HL-7702 cells. Empty pcDNA3.1 (-) plasmid was used as the mock-vehicle group. The $\mathrm{IC}_{50}$ values of tetrandrine in these four groups were compared. Overexpressed CYP2E1 in HL-7702 cells decreased the $\mathrm{IC}_{50}$ of tetrandrine (Figure 6C) and enhanced caspase 3 activity induced by tetrandrine (Figure 6D).

\section{Discussion}

In this study, we demonstrated that tetrandrine promotes mitochondrial dysfunction through ROS generated by CYP450, with CYP2E1 as the specific isoform that is primarily responsible for this process. Tetrandrine-induced cytotoxicity could be markedly reduced by ROS scavengers, indicating the major role of oxidative damage in tetrandrine-induced hepatotoxicity (Figure 2). Only CYP450 inhibitors could inhibit the tetrandrine-induced increase of ROS and GSH depletion (Figure 3), which implied the role of CYP450 in ROS production induced by tetrandrine. Additionally, an antioxidant (VC) and a CYP450 inhibitor (SKF-525A) could both prevent tetrandrine-induced mitochondrial dysfunction, although SKF-525A had a stronger protective effect (Figures 4 and 5). Finally, an inhibitor of the CYP2E1 isoform decreased the cytotoxicity and ROS production effects of tetrandrine. Overexpression of CYP2E1 enhanced the toxicity of tetrandrine (Figure 6). Taken together, these results suggest that the ROS from CYP450, spe-
A

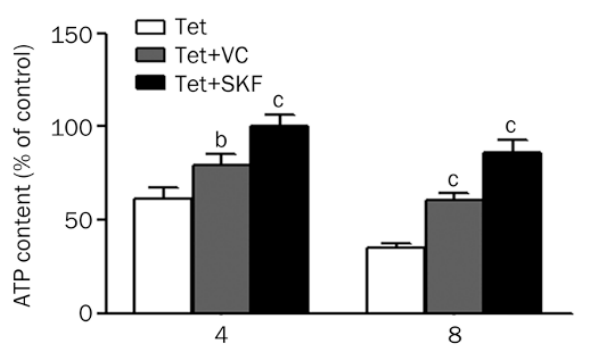

B
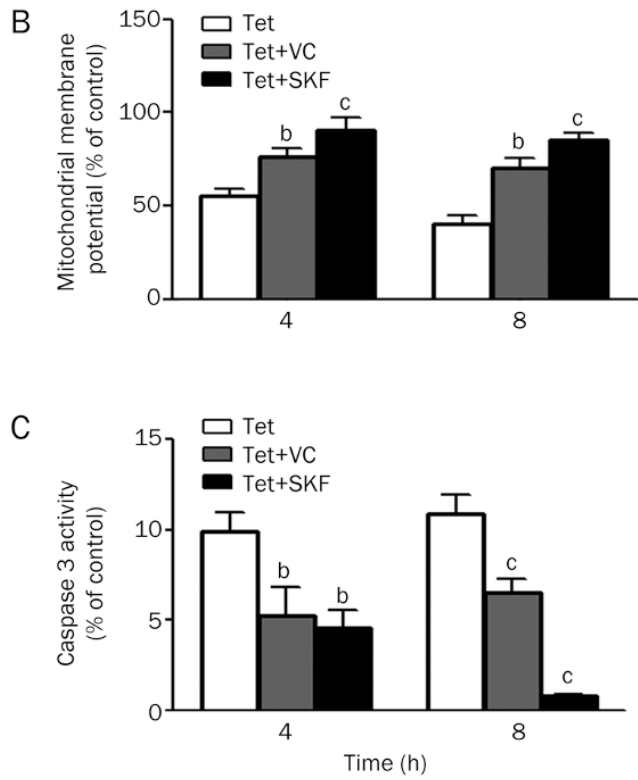

Figure 5. Protective effect of SKF and VC on mitochondrial dysfunction induced by tetrandrine. Rat hepatocytes were incubated with $25 \mu \mathrm{mol} / \mathrm{L}$ Tet, $10 \mu \mathrm{mol} / \mathrm{L}$ SKF and $1 \mathrm{mmol} / \mathrm{L}$ VC. (A) The effect of VC and SKF on tetrandrine-induced ATP depletion. Samples were taken at the indicated time points and assayed for ATP levels. (B) Effect of VC and SKF on mitochondrial membrane potential depletion induced by tetrandrine. (C) The activity of caspase 3 in the presence of tetrandrine and SKF. Caspase 3 activity was measured in rat primary hepatocytes at time-points up to 8 h. ${ }^{b} P<0.05,{ }^{c} P<0.01$ vs 'Tet' group at each time point. SKF, SKF$525 \mathrm{~A}$, a nonspecific inhibitor of CYP450. All results are presented as the mean \pm SD. $n=3$.

cifically CYP2E1, may account for the mitochondrial dysfunction induced by tetrandrine.

The CYP450 family of enzymes are responsible for the metabolism of both exogenous and endogenous materials ${ }^{[29]}$. Jin et al showed that inhibition of CYP3A attenuates the pulmonary toxicity induced by tetrandrine in mice, and only the human CYP3A4 and CYP3A5 isoforms are responsible for the reactive metabolites of tetrandrine ${ }^{[20]}$. In our study, ketoconazole and other CYP3A inhibitors had no effect on the cytotoxicity of tetrandrine, which may be due to the rapid decrease of CYP3A1/ 2 expression during the initial $24 \mathrm{~h}$ that rat hepatocytes are cultured on collagen ${ }^{[30]}$ and the ineffective inhibition of ketoconazole on rat CYP3A1/2 ${ }^{[31]}$.

A number of studies have shown the important role of 
A

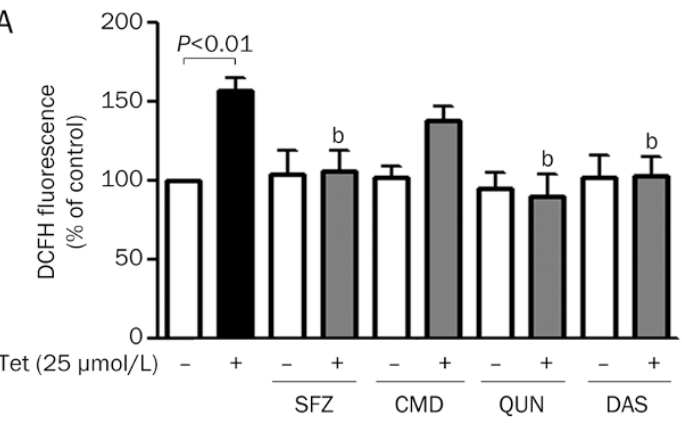

C

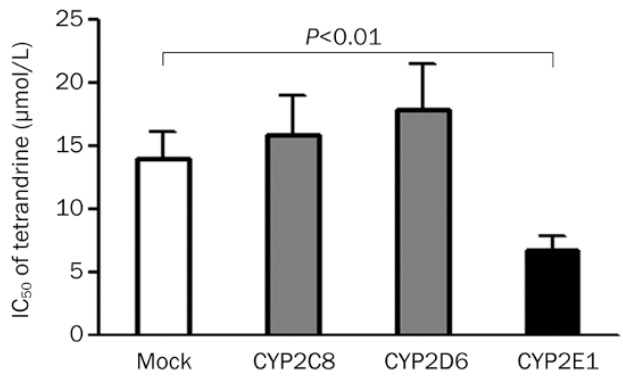

E

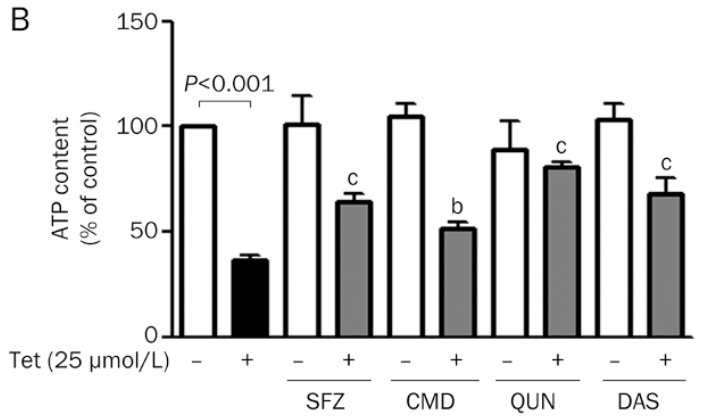

D

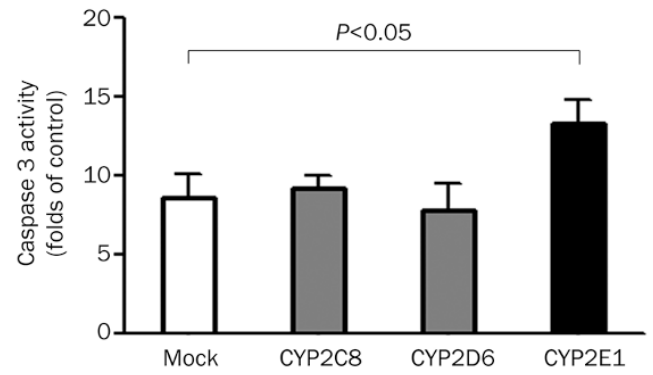

Figure 6. Role of CYP2E1 in tetrandrine-induced hepatotoxicity. (A) Effects of the CYP450 isoform inhibitors on the ROS production of tetrandrine. ROS was detected using the fluorescent probe DCFH-DA. Rat hepatocytes were incubated with $25 \mu \mathrm{mol} / \mathrm{L}$ Tet and the indicated inhibitors for $1 \mathrm{~h}$. The concentration of each inhibitor is listed in Table 2. (B) Effect of the CYP450 isoform inhibitors on the cytotoxicity of tetrandrine. Rat hepatocytes were treated with $25 \mu \mathrm{mol} / \mathrm{L}$ Tet for $8 \mathrm{~h}$. The concentration of each inhibitor was listed in Table 2. (C) IC ${ }_{50}$ values of tetrandrine in $\mathrm{HL}-7702$ cells overexpressing various CYP450 isoforms. Cells were treated with a range of tetrandrine concentrations $(0,0.03,0.1,0.3,1,3,10,30,100$, and 300 $\mu \mathrm{mol} / \mathrm{L}$ ). The $\mathrm{IC}_{50}$ values were calculated using the Logit method. (D) Caspase 3 activity in $\mathrm{HL}-7702$ cells overexpressing various $\mathrm{CYP} 450$ isoforms. Cells were treated with $25 \mu \mathrm{mol} / \mathrm{L}$ Tet for $8 \mathrm{~h}$. (E) The protein levels of CYP450 isoforms were evaluated by Western blotting. All of the results are presented as the mean \pm SD. $n=3 .{ }^{b} P<0.05,{ }^{c} P<0.01$ vs ' $25 \mu \mathrm{mol} / \mathrm{L}$ Tet' group.

overexpressed CYP2E1 in oxidative stress ${ }^{[32-37]}$. In this study, the protein levels of CYP2E1 in rat hepatocytes were well maintained (data not shown). Diallyl sulfide, an inhibitor of CYP2E1, decreased oxidative stress of tetrandrine and significantly attenuated ATP depletion induced by tetrandrine. Furthermore, overexpression of CYP2E1, but not CYP2C8 or CYP2D6, enhanced the toxicity and caspase 3 activity induced by tetrandrine in HL-7702 cells. Together, this strongly suggests a potential synergistic effect between CYP2E1 and tetrandrine in producing ROS. More work is necessary to clarify the mechanism through which CYP2E1 is involved in tetrandrineinduced hepatotoxicity.

In the present study, inhibitors of CYP450 largely decreased the ROS production induced by tetrandrine after $1 \mathrm{~h}$ (Figure 3A). However, CYP450 inhibitors did not completely recover the GSH depletion after $8 \mathrm{~h}$ (Figure 3B). It is well-known that GSH can be depleted by ROS and reactive metabolites of drugs. In a recent study, generation of the GSH conjugate from O-demethylated tetrandrine by CYP3A4 was observed with liver and lung microsomes. A high concentration of ketoconazole $(1 \mu \mathrm{mol} / \mathrm{L}$, approximately 50 -fold higher than the $K_{\mathrm{i}}$ value of ketoconazole on CYP3A4) did not completely inhibit the production of GSH conjugates (35\% GSH conjugates remained) (Figure S1 and Figure 9 in Ref 20) ${ }^{[20,31]}$, which was indicative of GSH consumption. SKF-525A has a much higher $K_{\mathrm{i}}$ value against CYP3A4 than ketoconazole, indicating less effective inhibition of CYP3A. Together, this suggests that the incomplete recovery of GSH depletion may be due to the persistent consumption of GSH conjugates with the metabolites of tetrandrine, which could not be completely inhibited by proadifen over a longer incubation time (Figure 3B).

In our previous study, daily administration of tetrandrine ( $57 \mathrm{mg} / \mathrm{kg}$ by oral gavage) for $8 \mathrm{~d}$ induced obvious liver injury in rats ${ }^{[9]}$. Early reports involving tetrandrine administration to rodents $(15 \mathrm{mg} / \mathrm{kg}$ by single gavage or $7.5 \mathrm{mg} / \mathrm{kg}$ by single intravenous injection) showed obvious accumulation in the 
rodent liver and very slow hepatic clearance. The concentration of tetrandrine in the liver reached approximately 22-32 $\mu \mathrm{mol} / \mathrm{L}$ and was maintained for at least $48 \mathrm{~h}^{[38,39]}$. Converting to an equivalent human dose, the dosage of tetrandrine for humans is approximately $2.38 \mathrm{mg} / \mathrm{kg}$. Clinical doses of tetrandrine range from 2 to $5 \mathrm{mg} / \mathrm{kg}$ over a course of approximately 3 months. Taken together, these data from the animal studies indicated the potential accumulation and injury to human livers from chronic administration of tetrandrine. Clinical attention regarding the dosage and treatment course of tetrandrine should be addressed.

In summary, ROS generated by CYP450 play a major role in tetrandrine-induced mitochondrial dysfunction, and CYP2E1 likely plays a more important role in this process. Our data will be helpful in the clinical application of tetrandrine.

\section{Acknowledgements}

This work was supported by National Natural Science Foundation of China (№ 30271558); Key projects of National Science and Technology Pillar Program (2012ZX09301001-006 and 2012zx09302003) and the Public Service Platform Project of Shanghai Science and Technology Committee (11DZ2292500).

\section{Author contribution}

Jin REN and Li-kun GONG participated in research design; Xin-ming QI, Ling-ling MIAO, and Yan CAI conducted experiments; Xin-ming QI, Ling-ling MIAO, and Li-kun GONG performed data analysis; Xin-ming QI, Ling-ling MIAO, Li-kun GONG, and Jin REN wrote or contributed to the writing of the manuscript.

\section{Supplementary information}

Supplementary information is available at Acta Pharmacologica Sinica website.

\section{References}

1 Xue Y, Wang Y, Feng DC, Xiao BG, Xu LY. Tetrandrine suppresses lipopolysaccharide-induced microglial activation by inhibiting NFkappaB pathway. Acta Pharmacol Sin 2008; 29: 245-51.

2 He FQ, Qiu BY, Li TK, Xie Q, Cui de J, Huang XL, et al. Tetrandrine suppresses amyloid-beta-induced inflammatory cytokines by inhibiting NF-kappaB pathway in murine BV2 microglial cells. Int Immunopharmacol 2011; 11: 1220-5.

3 Huang P, Xu Y, Wei R, Li H, Tang Y, Liu J, et al. Efficacy of tetrandrine on lowering intraocular pressure in animal model with ocular hypertension. J Glaucoma 2011; 20: 183-8.

4 Li X, Lu X, Xu H, Zhu Z, Yin H, Qian X, et al. Paclitaxel/tetrandrine coloaded nanoparticles effectively promote the apoptosis of gastric cancer cells based on "oxidation therapy". Mol Pharm 2012; 9: 222-9.

5 Liu C, Gong K, Mao X, Li W. Tetrandrine induces apoptosis by activating reactive oxygen species and repressing Akt activity in human hepatocellular carcinoma. Int J Cancer 2011; 129: 1519-31.

6 Shen DF, Tang QZ, Yan L, Zhang Y, Zhu LH, Wang L, et al. Tetrandrine blocks cardiac hypertrophy by disrupting reactive oxygen speciesdependent ERK1/2 signalling. Br J Pharmacol 2010; 159: 970-81.

7 Wei N, Liu GT, Chen XG, Liu Q, Wang FP, Sun H. H1, a derivative of Tetrandrine, exerts anti-MDR activity by initiating intrinsic apoptosis pathway and inhibiting the activation of Erk1/2 and Akt1/2. Biochem Pharmacol 2011; 82: 1593-603.

8 Wu CJ, Wang YH, Lin CJ, Chen HH, Chen YJ. Tetrandrine downregulates ERK/NF-kappaB signaling and inhibits activation of mesangial cells. Toxicol In Vitro 2011; 25: 1834-40.

9 Yan C, Xin-Ming Q, Li-Kun G, Lin-Lin L, Fang-Ping C, Ying X, et al. Tetrandrine-induced apoptosis in rat primary hepatocytes is initiated from mitochondria: caspases and endonuclease $G$ (Endo $G$ ) pathway. Toxicology 2006; 218: 1-12.

10 Tainlin L, Tingyi H, Changqi Z, Peipei Y, Qiong Z. Studies of the chronic toxicity of tetrandrine in dogs: an inhibitor of silicosis. Ecotoxicol Environ Saf 1982; 6: 528-34.

11 Li X, Su B, Liu R, Wu D, He D. Tetrandrine induces apoptosis and triggers caspase cascade in human bladder cancer cells. J Surg Res 2011; 166: e45-51.

12 Ding WX, Nam Ong C. Role of oxidative stress and mitochondrial changes in cyanobacteria-induced apoptosis and hepatotoxicity. FEMS Microbiol Lett 2003; 220: 1-7.

13 Pereira CF, Oliveira CR. Oxidative glutamate toxicity involves mitochondrial dysfunction and perturbation of intracellular $\mathrm{Ca}^{2+}$ homeostasis. Neurosci Res 2000; 37: 227-36.

14 Smith RA, Hartley RC, Cocheme HM, Murphy MP. Mitochondrial pharmacology. Trends Pharmacol Sci 2012; 33: 341-52.

15 Fernandes MA, Custodio JB, Santos MS, Moreno AJ, Vicente JA. Tetrandrine concentrations not affecting oxidative phosphorylation protect rat liver mitochondria from oxidative stress. Mitochondrion 2006; 6: 176-85.

16 Orrenius S, Gogvadze V, Zhivotovsky B. Mitochondrial oxidative stress: implications for cell death. Annu Rev Pharmacol Toxicol 2007; 47: 143-83.

17 Peng TI, Jou MJ. Oxidative stress caused by mitochondrial calcium overload. Ann N Y Acad Sci 2010; 1201: 183-8.

18 Vercesi AE, Kowaltowski AJ, Oliveira HC, Castilho RF. Mitochondrial $\mathrm{Ca}^{2+}$ transport, permeability transition and oxidative stress in cell death: implications in cardiotoxicity, neurodegeneration and dyslipidemias. Front Biosci 2006; 11: 2554-64.

19 Jang BC, Lim KJ, Paik JH, Cho JW, Baek WK, Suh MH, et al. Tetrandrine-induced apoptosis is mediated by activation of caspases and PKC-delta in U937 cells. Biochem Pharmacol 2004; 67: 1819-29.

20 Jin H, Li L, Zhong D, Liu J, Chen X, Zheng J. Pulmonary toxicity and metabolic activation of tetrandrine in CD-1 mice. Chem Res Toxicol 2011; 24: 2142-52.

21 Berry MN. High-yield preparation of morphologically intact isolated parenchymal cells from rat liver. Methods Enzymol 1974; 32: 625-32.

22 Orrenius S, Thor H, Rajs J, Berggren M. Isolated rat hepatocytes as an experimental tool in the study of cell injury. Effect of anoxia. Forensic Sci 1976; 8: 255-63.

23 Seglen PO. Preparation of rat liver cells. I. Effect of $\mathrm{Ca}^{2+}$ on enzymatic dispersion of isolated, perfused liver. Exp Cell Res 1972; 74: 450-4.

24 Wu EY, Smith MT, Bellomo G, Di Monte D. Relationships between the mitochondrial transmembrane potential, ATP concentration, and cytotoxicity in isolated rat hepatocytes. Arch Biochem Biophys 1990; 282: 358-62.

25 Mattia CJ, LeBel CP, Bondy SC. Effects of toluene and its metabolites on cerebral reactive oxygen species generation. Biochem Pharmacol 1991; 42: 879-82.

26 Dringen R, Hamprecht B. Glutathione content as an indicator for the presence of metabolic pathways of amino acids in astroglial cultures. 
J Neurochem 1996; 67: 1375-82.

27 Nieminen AL, Saylor AK, Tesfai SA, Herman B, Lemasters JJ. Contribution of the mitochondrial permeability transition to lethal injury after exposure of hepatocytes to t-butylhydroperoxide. Biochem J 1995; 307: 99-106.

28 Bradham CA, Qian T, Streetz K, Trautwein C, Brenner DA, Lemasters $\mathrm{JJ}$. The mitochondrial permeability transition is required for tumor necrosis factor alpha-mediated apoptosis and cytochrome $c$ release. Mol Cell Biol 1998; 18: 6353-64.

29 Andrade RJ, Robles M, Ulzurrun E, Lucena MI. Drug-induced liver injury: insights from genetic studies. Pharmacogenomics 2009; 10 : 1467-87.

30 Silva JM, Morin PE, Day SH, Kennedy BP, Payette P, Rushmore T, et al. Refinement of an in vitro cell model for cytochrome P450 induction. Drug Metab Dispos 1998; 26: 490-6.

31 Emoto C, Murase S, Sawada Y, Jones BC, Iwasaki K. In vitro inhibitory effect of 1-aminobenzotriazole on drug oxidations catalyzed by human cytochrome P450 enzymes: a comparison with SKF-525A and ketoconazole. Drug Metab Pharmacokinet 2003; 18: 287-95.

32 Caro AA, Cederbaum Al. Oxidative stress, toxicology, and pharmacology of CYP2E1. Annu Rev Pharmacol Toxicol 2004; 44: 27-42.
33 Leung TM, Nieto N. CYP2E1 and oxidant stress in alcoholic and nonalcoholic fatty liver disease. J Hepatol 2013; 58: 395-8.

34 Lee GH, Bhandary B, Lee EM, Park JK, Jeong KS, Kim IK, et al. The roles of ER stress and P450 $2 \mathrm{E} 1$ in $\mathrm{CCl}_{4}$-induced steatosis. Int $\mathrm{J}$ Biochem Cell Biol 2011; 43: 1469-82.

35 Aubert J, Begriche K, Knockaert L, Robin MA, Fromenty B. Increased expression of cytochrome P450 2E1 in nonalcoholic fatty liver disease: mechanisms and pathophysiological role. Clin Res Hepatol Gastroenterol 2011; 35: 630-7.

36 Kim HR, Lee GH, Cho EY, Chae SW, Ahn T, Chae HJ. Bax inhibitor 1 regulates ER-stress-induced ROS accumulation through the regulation of cytochrome P450 2E1. J Cell Sci 2009; 122: 1126-33.

37 Cho EY, Yun $\mathrm{CH}$, Chae HZ, Chae HJ, Ahn T. Anionic phospholipidinduced regulation of reactive oxygen species production by human cytochrome P450 2E1. FEBS Lett 2008; 582: 1771-6.

$38 \mathrm{Wu}$ JX, Gao LZ. Study in the pharmacokinetics of tetrandrine in vivo. Radiat Prot Bull 1982; 2: 29-37.

39 Li FQ, Lu B, Chen WB, Chao RB. Study on the distribution of lung targeted-tetrandrine sustained-release albumin microcapsules in vivo. Chin J Hospital Pharm 2001; 21: 259-61. 